\title{
Hacia una caracterización funcional de fijarse como marcador del discurso
}

\author{
JOSAPHAT ENRIQUE GUILLÉN ESCAMILLA \\ Profesor-investigador \\ Maestría en Diagnóstico y Rehabilitación Neuropsicológica \\ 3 Oriente No. 1413, \\ Barrio de Analco, Puebla, Pue. \\ C.P. 72000 \\ E-mail: josaphat.enrique@correo.buap.mx
}

\section{HACIA UNA CARACTERIZACIÓN FUNCIONAL DE FIJARSE COMO MARCADOR DEL DISCURSO}

RESUMEN: A partir de la propuesta teórica del Grupo Val.Es.Co., sobre unidades, posición y marcadores del discurso (Briz, A. y Pons, S., 2010; Grupo Val.Es.Co., 2014), el objetivo central de esta investigación es caracterizar las propiedades funcionales de la partícula discursiva $f i-$ jate/fijese. Para tal fin, revisamos la totalidad del Corpus Sociolingüístico de la Ciudad de México (Martín Butragueño, P. y Lastra, Y., 2011 2015) y analizamos las ocurrencias de fijate (que) / fijese (que) habilitadas con función discursiva. De acuerdo con los resultados, podemos adelantar que este marcador goza de una alta movilidad dentro de las unidades del discurso, por lo que desarrolla una amplia nómina de valores funcionales, principalmente asociados con la modalización, el control de contacto y la conexión. Finalmente, nuestros resultados apoyan la conclusión de que unidad y posición mantienen una estrecha relación en la atribución de funciones que pueden desempeñar los marcadores discursivos.

PALABRAS CLAVES: análisis del discurso; marcadores discursivos; unidades del discurso; modalización; control de contacto; conectores.

SUMARIO: 1. Introducción. 2. De verbos de percepción a marcadores discursivos. 3. Sobre unidades, posición y función. 4. Metodología. 5. Análisis. 5.1. Enfocador de la alteridad. 5.2. Focalizador. 5.3. Introductor de intervención reactiva. 5.4. Intervención con valor fático. 5.5. Introductor de discurso directo citado. 5.6. Cambio de tópico. 6. Conclusiones.

Fecha de Recepción Fecha de Revisión Fecha de Aceptación Fecha de Publicación

\section{TOWARDS A FUNCTIONAL CHARACTERIZATION OF FI- JARSE AS A DISCOURSE MARKER}

ABSTRACT: Beginning with the theoretical framework proposed by Grupo Val.Es.Co., about units, position, and discourse markers (Briz, A. \& Pons, S., 2010; Grupo Val.Es.Co., 2014), the central aim in this research is to characterize functional properties of discursive particle fijate/fijese. To achieve this aim we analyse the data from Corpus Sociolingüístico de la Ciudad de México (Martín Butragueño, P. y Lastra, Y., 2011-2015). We analyze occurrences of fijate (que) / fijese (que) when functions as discourse marker. According to our results, we claim that this marker has a high mobility within discursive units, so that it develops a wide range of functional values, mainly associated with modalization, contact's control, and connection. Finally, our results support the idea that discursive units and positions play a key role in the attribution of functions that can be performed by discourse markers.

KEY WORDS: discourse analysis; discourse markers; units of discourse; modalization; contact's control; connectors.

SUMMARY: 1 . Introduction. 2 From perception verbs to discourse markers. 3. About units, positions, and functions. 4. Methodology. 5. Analysis. 5.1. Focalizer of the alterity. 5.2. Focalizer. 5.3. Reactive intervention introducer. 5.4. Intervention with phatic value. 5.5. Direct speech introducer. 5.6. Topic change. 6. Conclusions.

\section{VERS UNE CARACTERISATION FONCTIONNELLE DU FIJARSE EN TANT QUE MARQUEUR DU DISCOURS}

RÉSUMÉ: A partir de la proposition théorique du Grupo Val.Es.Co., sur les unités, la position et les marqueurs du discours (Briz, A. y Pons, S., 2010; Grupo Val.Es.Co., 2014), l'objectif principal de cette recherche est de caractériser les propriétés fonctionnelles de la particule discursive fijate/fijese. À cette fin, nous avons revu la totalité du Corpus Sociolingüístico de la Ciudad de México (Martín Butragueño, P. y Lastra, Y., 2011$2015)$ et nous avons analysé les apparitions de fijate (que) / fijese (que) dans leur dimensión discursive. Selon les résultats, nous pouvons avancer que ce marqueur discursif jouit d'une grande mobilité au sein des unités discursives, il développe donc un large éventail de valeurs fonctionnelles, principalement associées à la modalisation, au contrôle des contacts et à la connexion. Enfin, nos résultats permettent d'affirmer que l'unité et la position maintiennent une relation étroite dans l'attribution des fonctions que peuvent remplir les marqueurs discursifs.

MOTS CLÉS: analyse du discours; marqueurs discursifs; unités du discours; modalisation; contrôle de contact; connecteurs.

SOMMAIRE: 1. Introduction. 2. Des verbes de perception aux marqueurs discursifs. 3. A propos d'unités, position et fonction. 4. Méthodologie. 5. Analyse. 5.1. Focalisateur de l'altérité. 5.2. Focalisateur. 5.3. Introducteur d'intervention réactive. 5.4 Intervention à valeur phatique. 5.5 Introducteur du discours directe. 5.6 Changement de topique. 6 . Conclusions. 


\title{
Hacia una caracterización funcional de fijarse como marcador del discurso
}

\author{
JOSAPHAT ENRIQUE GUILLÉN ESCAMILLA
}

\section{INTRODUCCIÓN}

Las investigaciones sobre verbos de percepción habilitados como marcadores discursivos cuentan ya con una amplia tradición dentro de los estudios pragmáticos y discursivos hispánicos (Beinhauer, 1991 [1929]; Alcina, J. y Blecua, J. M., 1975; Fuentes Rodríguez, 1990; Pons, 1998, 2002; Cuenca, M. J. y Marín, J. M., 2000; Galué, 2002; Gallardo-Paúls, B. y Jordà, M. J., 2005; Briz, A. y Pons, S., 2010; Tanghe, S. y Jansegers, M., 2012). La mayoria de estos autores consideran que, dado que este tipo de verbos proviene de un mismo grupo semántico plenamente identificado -el de la percepción sensorial-, comparten una serie de características que permiten establecer sus rasgos formales y funcionales de manera precisa: (i) tienen un carácter parentético, esto es, tienden a aparecer en la periferia del enunciado, principalmente en la periferia izquierda; (ii) son formas imperativas o relacionadas con el imperativo; (iii) tienen un valor conativo básico que las vincula con el receptor, comunicativamente, y, por ende, a la segunda persona (tú o usted), por lo que no son completamente invariables desde el punto de vista morfológico y conservan la flexión para la segunda persona de cortesía1; (iv) tienen valores relacionados con la llamada de atención, asociados con la gestión de la interacción, por lo que (v) son formas típicas de la lengua oral (Cuenca, M. J. y Marín, J. M., 2000: 216; Gallardo-Paúls, B. y Jordà, M. J., 2005: 60).

En este sentido, la atención ha recaído en las formas más típicas de estos verbos: a ver, mira, oye, escucha, ve, prestando menos atención a la forma fijjate/fïjese o tratándola solo de manera periférica o parcial (cf. Galué, 2002; Domínguez, C. L. y Álvarez, A., 2005), tal vez por su menor frecuencia con respecto a los otros marcadores. Así pues, el estudio de esta partícula no ha sido del todo exhaustivo, por lo que el objetivo de este trabajo es caracterizar las funciones que puede desempeñar, atendiendo principalmente a la unidad de la que forma parte y la posición en la que aparece. Finalmente, queremos hacernos eco de la propuesta de Briz y Pons sobre la relación entre marcadores discursivos y posición, fundamental para nuestro análisis, ya que "la variación funcional de los marcadores del discurso está limitada por su posición discursiva y por el tipo de unidad en que se integra. Ello quiere decir que [...] la unidad y la posición están en correlación en gran medida

\footnotetext{
${ }^{1}$ Autores como Pons (2002) indican que sólo se mantienen aquellas diferencias de número, cuando hay más de un interlocutor, y principalmente las diferencias pragmáticas de cortesía, esto es, la alternancia entre tú y usted.
} 
con la función de los marcadores discursivos" (Briz, A. y Pons, S., 2010: 327).

\section{DE VERBOS DE PERCEPCIÓN A MARCADORES DISCURSIVOS}

En los estudios previos (Cuenca, M. J. y Marín, J. M., 2000; Galué, 2002; Gallardo-Paúls, B. y Jordà, M. J., 2005; Pons, 2008; Tanghe, S. y Jansegers, M., 2012; Fernández Jaén, 2012) ha quedado muy bien establecido cuál ha sido el proceso de gramaticalización que han seguido estos verbos de percepción para habilitarse como marcadores discursivos. Este proceso supone una pérdida del significado léxico que favorece la asunción de una o más funciones discursivas, esto es, estas unidades léxicas pasan de tener un valor predicativo a tener uno discursivo y, por otro lado, también se produce un cambio de categoria, de verbo a interjección. Esto es posible gracias a que estas formas pierden varios de los rasgos que definen al verbo como categoría (Pons, 2002: 216), pero, por estar en modo imperativo, conservan un valor fático y conativo. En otras palabras, debido a su origen imperativo, cumplen con "una función intersubjetiva, orientada hacia el receptor, por lo que $[. .$.$] son utilizadas para invitar al interlocutor a que mantenga su aten-$ ción durante la intervención del hablante" (Gallardo-Paúls, B. y Jordà, M. J., 2005: 84).

Ahora bien, los verbos de percepción visual -en contraste con los de percepción auditiva- "manifiestan una subjetivización mayor, puesto que, en su uso discursivo, han perdido totalmente la referencia al sentido de la vista" (Cuenca, M., J. y Martín, J. M., 2000: 224), por lo que los hablantes tienden a usar, por defecto, el marcador derivado de un verbo de percepción con una pronunciada polisemia ${ }^{2}$. En consecuencia, mira/mire y ve/vea suelen ser utilizados con mayor frecuencia que oye/oiga y escucha/escuche.

Tanto oye como mira poseen un significado literal que, en imperativo, consiste en una invitación a mirar o a oír [...] En términos más técnicos, se puede decir que [este] valor fático es una instrucción convencional y no conversacional. Sin embargo, el caso de mira es distinto, ya que el significado literal consiste en una invitación a mirar un elemento de la enunciación (Pons, S., 2002: 215).

Así pues, gracias a su origen, el uso de estas formas está basado en las funciones fática y conativa del lenguaje, y es justamente esta herencia la

\footnotetext{
2 En una escala de gramaticalización, los extremos corresponden prototípicamente a a ver [+gramaticalizado] y a oye [-gramaticalizado], mientras que en la parte media se encuentran mira y fijate. De acuerdo con Gallardo-Paúls y Jordà (2005), en el primer caso las funciones están orientadas hacia el emisor [+subjetivo], en tanto que, en el segundo caso, están orientadas hacia el receptor [+intersubjetivo]. En este sentido, el marcador tendrá una polisemia más pronunciada cuando cuente con una entrada más amplia (Tanghe, S. y Jansegers, M., 2012), esto es, mientras esté más cercano a los rasgos: [+agentividad], [+volición], [+intencional], [+finalidad] y [+movimiento].
} 
que detona el cambio semántico y todas las demás modificaciones estructurales que trae consigo el cambio de categoría (Cuenca, M. J. y Marín, J. M., 2000).

Como es habitual, el cambio de categoría trae consigo nuevos matices morfológicos, sintácticos y fonéticos. En el primer caso, pierden la alternancia de morfemas flexivos que se limita únicamente a la segunda persona del singular, sea de cortesía (usted) o de familiaridad (tú). En cuanto a su engarce sintáctico en la oración, se observa que las unidades que anteceden o suceden a estas formas "no pueden ser interpretadas como constituyentes de un sintagma verbal o como complementos subcategorizados del mismo" (Pons, 2002: 217), ya que no pueden ser sustituidas por clíticos pues, al hacerlo, la función de estos verbos cambia por completo, desde una perspectiva discursiva. En cuanto a su fonética, su estructura también se ve afectada; en particular se ha observado que presentan una reducción fónica, como en los casos de $(m)$ ira o los de $f i(j)$ ate, rasgo que refuerza la explicación de cambio de categoria. Finalmente, en cuanto a la entonación, no suelen estar integrados en la línea melódica del enunciado al que pertenecen.

Con respecto a la organización textual, Gallardo-Paúls y Jordà (2005: 71) sostienen que su función es la de llamar o mantener la atención del receptor, en estos casos comúnmente se introducen "fragmentos de discurso directo, pero también se pueden utilizar como elementos modalizadores y como formas que dan paso a comentarios de tipo personal o microrelatos y explicaciones". Con esta función modalizadora se utilizan para dar mayor expresividad a la narración y el hablante trata de comprometer al interlocutor con lo comunicado (Vigara Tauste, 1980). Pragmáticamente, gracias a su origen, se caracterizan por la fuerza apelativa que poseen y "por marcar una reorientación de la progresión del discurso, básicamente en dos niveles: distribución del turno de habla y desarrollo del tema. En este sentido, suelen indicar una pausa o un corte en la comunicación en curso, con una intención a menudo argumentativa" (Cuenca, M. J. y Marín, J. M., 2000: 232). Finalmente, desde una perspectiva argumental, pueden marcar una oposición a un argumento que otro hablante esté defendiendo; también pueden interrumpir el flujo de la conversación y pedir un cambio, ya sea de emisor, receptor, de orientación argumentativa o de tema.

En resumen, este proceso de gramaticalización habilita a estos verbos de percepción visual como marcadores del discurso, que desempeñan funciones particulares de organización textual, modalizadoras, argumentativas y de control de contacto (como signo de alteridad); además, trae consigo cambios formales en el plano fonético, morfológico y sintáctico. Ahora bien, lo que nos interesa analizar aquí es si todas estas funciones también pueden ser desempeñadas por fíjate y/o si este marcador cumple algunas otras particulares. Para tal fin, necesitamos revisar dos aspectos que han demostrado ser determinantes al describir las funciones de estas partículas discursivas: la unidad en la que aparecen y la posición del marcador. 


\section{SOBRE UNIDADES, POSICIÓN Y FUNCIÓN}

El sistema de unidades propuesto por el Grupo Val.Es.Co. (Briz, 1998, 2000, 2006; Briz, A. y Grupo Val.Es.Co., 2002, 2003; Briz, A. y Pons, S., 2010; Grupo Val.Es.Co., 2014), a partir del modelo de la Escuela de Ginebra (Roulet, et al., 1985, 2001), se revela como una herramienta de análisis dispuesta, entre otras cosas, para el estudio de los marcadores discursivos, atendiendo principalmente al tipo de unidades que conforman o del que son parte, así como a la posición que tienen en la conversación, ya que esto, en conjunto, dota de matices funcionales a estas partículas discursivas.

Este sistema está conformado por ochos unidades (discurso, diálogo, intercambio/alternancia de turnos, intervención/turno, acto y subacto), donde la conversación tiene como límite superior el discurso, cuya unidad mínima es el intercambio/alternancia de turnos, y como límite inferior el acto y subacto, que componen la unidad máxima monológica, la intervención/turno. Luego, la unión de dos intervenciones de distintos emisores constituye un intercambio, que puede estar formado por una intervención iniciativa -directa o indirecta- (aquella que intenta provocar o provoca habla posterior) y otra reactiva -cooperativa, evaluadora, fática o completiva- (la respuesta o reacción) o compuesta (consta de una intervención que es reactiva e iniciativa a la vez).

En este escenario, se plantean tres dimensiones para distinguir entre tipos de unidades. La primera es la dimensión estructural -de naturaleza interna- que señala la forma en que los constituyentes se suceden para construir un mensaje. La segunda es la dimensión social -de naturaleza externaque está relacionada con la influencia que ejercen los participantes sobre la estructura resultante. Finalmente, la dimensión informativa muestra la manera en que se divide un mensaje (Grupo Val.Es.Co., 2014: 15-6). Esta división ayuda a distinguir entre unidades de indole estructural y unidades de indole social, por eso la intervención goza solo de un carácter estructural interno, esto es, corresponde únicamente a la producción de material fónico por parte de un emisor -corresponderia a la fuerza locutiva-, a diferencia del turno que está revestido, además, de un carácter social externo, pues influye en el desarrollo temático de la conversación:

\footnotetext{
En función de estos dos criterios (cambio de emisor y contribución a la conversación) estableceremos una diferencia entre dos tipos de unidades: las primeras se denominan intervenciones y vienen delimitadas prototípicamente por el cambio de emisor [...] Las segundas, además, de por dicho cambio, se reconocen por efectuar una contribución positiva al desarrollo de la conversación y se denominarán turnos (Grupo Val.Es.Co., 2014: 17)
}

El lector se habrá percatado de la relación de inclusión unidireccional que se establece: todo turno se constituye, al mismo tiempo, como intervención, pero no toda intervención será un turno. 
Así pues, la intervención corresponde a la emisión de un hablante y puede estar conformada por uno o más actos. Estos actos son unidades que representan per se una acción comunicativa y, en consecuencia, son aislables, es decir, cada acto puede constituirse como una intervención. Finalmente, los subactos son las unidades informativas minimas en las que puede quedar dividido un acto y, de acuerdo con su valor, pueden ser clasificados en: (i) subactos sustantivos directores (SSD, núcleo proposicional del acto en el que aparecen y portador de la fuerza ilocutiva del acto), (ii) subactos sustantivos subordinados (SSS, semántica e informativamente supeditados a los directores), (iii) subactos subordinados topicalizados (SSSTop, formados por elementos desplazados a la derecha o a la izquierda por ser informativamente más relevantes), (iv) subactos adyacentes textuales (SAT, organizan y distribuyen el flujo del habla), (v) subactos adyacentes modalizadores (SAM, señalan algún tipo de matización específica, ya sea atenuación o intensificación), y (vi) subactos adyacentes interpersonales (SAI, implican la interacción entre hablante y oyente) (Pons, 2008; Briz, A. y Pons, S., 2010, Grupo Val.Es.Co., 2014).

A partir de estas distinciones, se refinan las nociones de posición inicial, intermedia y final, ya que ahora serán consideradas con respecto a la unidad discursiva y a la posición que ocupa el marcador discursivo dentro de la unidad en la que se encuentra, ya sea intervención, acto o subacto; así, será más exacto decir posición inicial de diálogo, posición inicial de intervención, posición inicial de acto o posición inicial de subacto. Lo mismo se aplica para las otras posiciones. Finalmente, si consideramos tanto el tipo de unidad que conforman o del que son parte, así como la posición que guardan, el análisis de las funciones de los marcadores discursivos será más productivo y detallado, ya que ambos factores dotan de matices funcionales a estos elementos.

\section{Metodología}

Para nuestro estudio recurrimos al análisis del Corpus Sociolingüístico de la Ciudad de México (CSCM, Martín Butragueño, P. y Lastra, Y., 20112015). En total, el CSCM está compuesto por 108 entrevistas divididas en tres grupos de acuerdo con el nivel de instrucción3: alto (36), medio (36) y bajo (36). Estos, a su vez, están subdivididos en tres grupos etarios: jóvenes (20-34), adultos (35-54) y mayores (55 en adelante). Finalmente, los informantes están agrupados por género. Los temas generales de las entrevistas giraron en torno al trabajo, la familia y el tiempo libre del informante, por lo

\footnotetext{
${ }^{3}$ El nivel más bajo incluye a las personas que han llegado a concluir la primaria, de tal forma que recibieron hasta 6 años de escolarización. En este nivel también están incluidos los analfabetos y quienes no terminaron la primaria. El nivel medio se compone por las personas que cursaron hasta la enseñanza media, o sea, personas que recibieron hasta 12 años de escolaridad. Finalmente, el nivel alto incluye a hablantes con enseñanza superior, es decir, recibieron al menos 16 años de escolaridad. Este grupo puede incorporar informantes con estudios de posgrado.
} 
que los tópicos fueron, hasta cierto punto, parecidos en todos los informantes (Martín Butragueño, P. y Lastra, Y., 2011-2015).

A pesar de que este no es un estudio de corte sociolingüístico, decidimos utilizar el CSCM debido a que el estilo empleado en las entrevistas oscila entre dos polos: (i) la entrevista semiinformal [+formal], caracterizada por la tendencia a emplear la variante formal, y (ii) la conversación grabada [-formal], que llega a mostrar, incluso, pocas diferencias con respecto a la conversación coloquial entre conocidos. De acuerdo con Martín Butragueño y Lastra, fue común que algunas entrevistas comenzaran por presentar una estructura semiinformal, pero a lo largo de su desarrollo terminaran en conversaciones.

Así pues, tomamos el total de las entrevistas (108) y, a partir de las transcripciones disponibles en linea4, comenzamos el rastreo de las apariciones de fijate/fijese y nos quedamos solo con aquellas en las que se empleaban como marcadores discursivos. Una vez hecho esto, procedimos a analizar las funciones que desempeñaban atendiendo principalmente al tipo de unidad en el que aparece y la posición de la partícula discursiva. Finalmente, presentamos los ejemplos con las convenciones originales de etiquetado PRESEEA $^{5}$ (2008), salvo en los casos que la posición del marcador no es transparente y necesita una segmentación más exacta. En tales casos seguimos las convenciones propuestas en Pons (2016) para la división de la conversación en actos y subactos.

\section{ANÁLISIS}

\subsection{ENFOCADOR DE LA ALTERIDAD}

Como vimos en el apartado 2, gracias a su origen -el del acto físico de fijarse-, este marcador desarrolla una función centrada en llamar la atención del oyente, por lo que ha sido descrito como un marcador de interacción, enfocador de alteridad o fático (Beinhauer, 1991; Tanghe, S. y Jansegers, M., 2012; Galué, 2002; Pons, 1998). Es utilizado para interpelar al interlocutor y llamar su atención. Por lo general, introduce o remite a una información que se dará a continuación y de la que se desea hacer partícipe al oyente para acercarlo a ámbito del hablante (Galué, 2002: 39), por lo que tiene un carácter fórico. Con esta función, el marcador goza de cierta movilidad puesto que puede presentarse a inicio de intervención o de acto:

[Hablando sobre los estudios y la escuela]

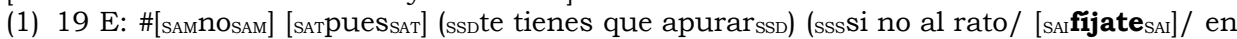

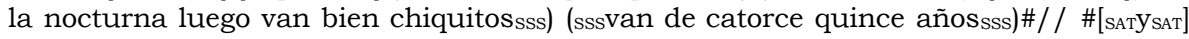

\footnotetext{
4 En linea. Disponible en: "http://lef.colmex.mx/index.php/investigaciones/corpussociolingueistico-de-la-ciudad-de-mexico-cscm".

${ }^{5}$ El Proyecto para el Estudio Sociolingüístico del Español de España y América es un proyecto para la creación de un corpus panhispánico de lengua hablada, atendiendo a la variedad geográfica y social.
} 


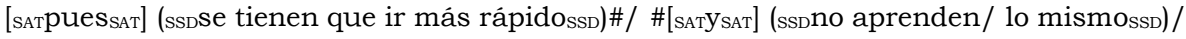
(ssDaprenden regularssD)/ (ssspor eso apúrate de una vez ahoritasss)\# (entrevista 92)

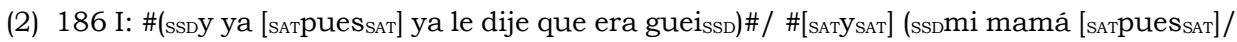
[sAI fijate $_{\mathrm{SAI}}$ ]/ nunca me hubiera esperado una/ reacción así de mi mamássD)\# (entrevista 42)

(3) 383 E: \#ah\#/ \#[saloye $\left.{ }_{\mathrm{SA}}\right] /$ (ssD ¿iy sí le gustó a tu amiga lo de este del/ del amigo se-

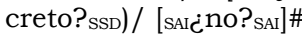

384 I: \#[sAIfijate que ${ }_{\mathrm{SAI}}$ ] (ssDno le preguntésSD)\# (entrevista 46)

Así pues, este uso está relacionado principalmente con la gestión de la interacción, por lo que comúnmente se presenta como subacto dentro de una intervención, como en (1) y (2) -donde se encuentra encajado dentro de un subacto sustantivo-, o a inicio de intervención reactiva (ejemplo 3). En estos casos, el marcador se constituye como un subacto adyacente interpersonal (SAI), por lo que la partícula discursiva tiene un valor perlocutivo (Pons, 1998) o conativo (Jakobson, 1981), con el que el hablante busca la reacción del oyente o influir en él. Finalmente, cabe mencionar que esta es la función típica de este marcador y la que más se ha descrito. Por ejemplo, Galué (2002: 39) concluye que fíjate/fíjese "se limita a la función apelativa y catafórica con la que el hablante introduce enunciados cuya información desea compartir con su interlocutor". O, como sostienen Domínguez y Álvarez (2005: 6), "es una forma apelativa dirigida al interlocutor y presentada en la correspondiente segunda persona gramatical". No obstante, esta partícula desempeña varias funciones más, como veremos a continuación.

\subsection{FOCALIZADOR}

Existen otros ámbitos del valor fático descrito anteriormente. En particular, nos referimos a lo que Pons (1998) ha denominado función fática interna, donde, ahora, el marcador dirige la atención a una parcela discursiva específica. Cuando es el caso, el llamado de atención recae sobre el enunciado y no sobre la enunciación, por tal motivo el constituyente que introduce fijate/fijese tiende a presentar información no esperada o una contraexpectactiva.

[Hablando sobre un permiso para poner una tienda]

(4) 1712 I: \#[sATO seasat] (ssda mí m- yo creo me anda costando unos mil pesos [saíno? permiso a lo mejor $\left.[<q u e>]_{\text {ssd }}\right) \#$

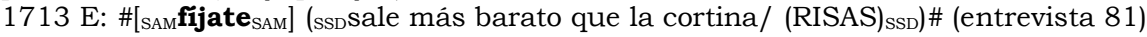

(5) $854 \mathrm{E}:$ \#[ah]\#/ \#(ssD¿̉cuántos años tiene su nieta?ssD)\#

855 I: \#(ssDtreinta ssD \#

$856 \mathrm{E}:$ \#(ssditreinta ya!ssD)\#

857 I: \#(ssDtreintassD)\# \#[satysaT] (ssDla otra tiene/ co- tendrá como veintiséis o veinticin$\left.\mathrm{CO}_{\mathrm{SSD}}\right) \#$

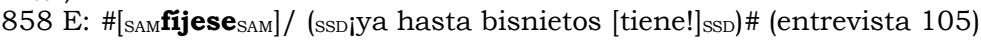


En (4) y (5), el marcador se encuentra a inicio de intervención y también se presenta como un subacto, pero en este caso como uno adyacente modalizador. En estos ejemplos, el marcador dirige la atención -de forma prospectiva- a una parcela discursiva particular, en (4) destacando que resulte más barato tramitar un permiso que la cortina del local, mientras que en (5) realza el hecho de que el interlocutor sea ya bisabuelo, lo que se nota aún más claro con la aparición del focalizador aditivo hasta, que confiere al constituyente que introduce fijate un carácter inesperado (Briz, A., Pons, S. y Portolés, J., 2008).

Ahora bien, a pesar de no ser la posición preferida del marcador, también puede aparecer a final de acto o de intervención desempeñando la misma función, pero con un valor anafórico de refuerzo:

(6) $441 \mathrm{I}:$ \#(ssphay un tianguis ahí [también $\mathrm{mm}]_{\mathrm{ssD}}$ )\#

$442 \mathrm{E}:$ \#(ssD[también viene el tianguis] $\left.]_{\mathrm{ssD}}\right) \#$

443 I: \#(ssd qué día?ssd)\#

$444 \mathrm{E}:$ \#(ssDviene los/ miércoles y los viernesssD)\#

445 I: \#(ssDlos viernes ssD \#

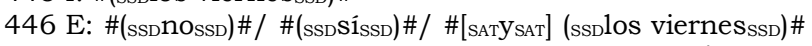

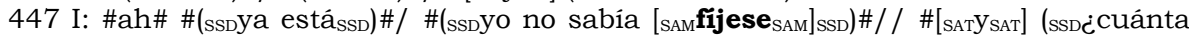
gente hay que se dedica a barrer aqui en la unidad?ssD)\# (entrevista 87)

[Hablando de la Revolución Mexicana]

(7) 182 I: \#(ssdasí eso eso fue 1- la razón de mil novecientos/ de lo de la/ pasando la Revolución ${ }_{\mathrm{SSD}}$ )\# \#[SATentonces $\mathrm{SAT}_{\mathrm{SA}}$ (ssmis padres me comentaban que era ¡muy! triste triste)/ demasiadossd) (sssporque no tien- no tenían ni qué comersss)\#

183 E: \#[híjole]\#

184 I: \#(ssD[el que] aguantaba el hambre bien $\left.{ }_{\mathrm{SSD}}\right) \#$ \#[SATYSAT] (ssDel que no se moría ${ }_{\mathrm{SSD}}$ )\# $185 \mathrm{E}:$ \#(ssDटंa poco?ssD)\#

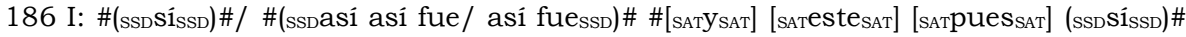
\#[satysat] (ssdde alli para acá/ después de la Revolución se medio mejoróssD)/ (ssspero ahora de nuevo va empezando [sam fijese SAM $\left._{\text {Sss }}\right) \#$ (entrevista 97)

En (6) y (7), el marcador refuerza la importancia comunicativa de la unidad discursiva recién presentada, en el primer caso "yo no sabía", mientras que en el segundo "de nuevo va empezando". Así pues, fijate funciona como una marca lingüística de relevancia -prospectiva o retrospectiva- que hace las veces de guía en el procesamiento del enunciado en el que se encuentra (Pons, 1998: 220), ya que lo reviste con una mayor prominencia comunicativa, garantizando así su relevancia óptima (Sperber, D. y Wilson, D., 1986).

Finalmente, hay que decir que esta posición fue común en el corpus, por lo que fijate/fijese desarrolla valores fóricos tanto prospectivos como retrospectivos, asociados con la libertad de posición de la que goza. En este mismo sentido, y en comparación con otros marcadores parecidos, esta partícula parece tener un carácter anafórico más natural: 
(8) Vine porque la prensa mintió. Dijeron que abririan las taquillas el martes y mira [siguen cerradas] [no sucedió] (tomado del DPDE6).

En el ejemplo (8), después del marcador hay estructura elidida o sobreentendida ${ }^{7}$, por lo que propiamente dicho no se encontraria a final de intervención; además, en estos casos parece que el marcador guarda una mayor cercanía al acto físico de mirar. Y esto no parece ser el caso de fijate:

(9) 230 I: \#(sspen diciembre me di cuenta que estaba < yo> embarazadassD)\# \#[satysaT] (ssdije bueno $\left._{\mathrm{SSD}}\right) \#$ / \#(ssDya tenía tres meses de embarazo [sam fijate $\left.\left._{\mathrm{SAM}}\right]_{\mathrm{SSD}}\right) \# /$ \#(ssDyo no tenía ascos ni mareosssD)\# (entrevista 57)

Así, en (9), gracias a la posición final de acto, el marcador está intensificando la información recién presentada: "ya tenía tres meses de embarazo"; además, la pausa posterior al marcador confirma que es un subacto adyacente modalizador que tiene un alcance fórico retrospectivo. Con todo, mira $\mathrm{y}$ oye también pueden aparecer a final de acto o intervención, pero teniendo otros matices como el desacuerdo ( $c f$. Briz, A. y Pons, S., 2010).

\subsection{INTRODUCTOR DE INTERVENCIÓN REACTIVA}

Distribucionalmente, fijate tiene una predisposición para aparecer a inicio de intervención reactiva, es decir, desarrolla una función de introductor de respuestas (Briz, 1993b: 45; Martín Zorraquino, M. A. y Portolés, J., 1999: 4084). Así, es muy frecuente que fijate/fíjese sean utilizados como introductores de intervenciones reactivas a preguntas, peticiones o aseveraciones, con lo que adquieren una dimensión social y se convierten en turnos porque "consiguen hacer avanzar la conversación" (Grupo Val.Es.Co., 2014: 22).

Así pues, gracias a esta tendencia de aparecer a inicio de intervención, este marcador también viene acompañado de un matiz modalizador, ya que su valor catafórico puede: (i) adelantar una justificación (ejemplo 10) o (ii) presentar una respuesta despreferida (ejemplos 11 y 12). En resumen, tiene un papel conversacional como marcador de respuesta ${ }^{8}$.

(10) $427 \mathrm{E}$ : y te iba a preguntar ¿qué tal te llevas con tus cuñados?

428 I: fijate al principio al principio sí me < me:>/ tenía yo el celo $429 \mathrm{E:}$ ¿sí? [(risa)]

430 I: [sí sí pasa] sí luego sí/ tenía el celo de mi hermana no pues < pus> "quihúbole $<\sim$ queóbole> pues < pus>/ se va a llevar a mi [hermana] (entrevista 85)

[Hablando de comprar ropa]

(11) 1105 E: pero bueno también/// pues// bueno M sí ya está en la edad de que quiere

\footnotetext{
${ }^{6}$ Diccionario de Partículas Discursivas del Español (Briz, A., Pons, S. y Portolés, J., 2008).

${ }^{7}$ Estaria asociado a lo que Gallardo-Paúls (2013) ha denominado generador de inferencias, provocado precisamente por la aparición del marcador.

8 Esta función es muy parecida a la de pues cuando se encuentra a inicio de intervención reactiva. Cf. Briz, 1993a.
} 
más ropa/ ¿no?/ P/

1106 I: fijate que < que:>/ no tanto así de < de:> vestid- bueno sí/ pero sí la < la:>/ la la restrinjo un poquito (entrevista 69)

(12) $661 \mathrm{E}:$ ¿y cuáles fueron los hoteles seleccionados?/ seguro los de Tlalpan 662 P: [mh]

663 I: [fijate] que de Tlalpan/ no/ (entrevista 21)

En (10), el hablante presenta su opinión y justifica por qué al principio tenía una mala relación con sus cuñados. En estos casos, el marcador gracias a su valor fático- invita al oyente a aliarse a la opinión del hablante o, al menos, acercarlo a su ámbito. Además, aquí, el marcador desempeña un papel modalizante muy parecido al de es que, que "conecta dos enunciados y, además, es un índice de la presencia del hablante, a partir del cual se justifica algo" (Briz, 1993a: 155). En cuanto a (7) y (8), fijate que introduce una respuesta despreferida -en claro contraste con la opinión vertida por el interlocutor- al tiempo que se destaca la información que se presenta por ser menos esperada.

\subsection{INTERVENCIÓN CON VALOR FÁTICO}

Ya ha quedado más o menos claro que fijate tiene un amplio rango de libertad de posición. En este sentido, la muestra máxima de independencia de esta partícula discursiva se refleja cuando constituye, por sí misma, una intervención, en cuyo caso cumple con una función fática, es decir, no contribuye al desarrollo efectivo de la conversación, más bien sirve para mantener o verificar el canal de la comunicación (Jakobson, 1981):

[Hablando sobre la desmejorada salud de un conocido]

(13) 399 I: su cara/ su cabeza/ tenía ya su pelo blanco < bla:nco> como algodón/ todo y sus cabellos para < pa> arriba/ ya estaba como/ a razón de cinco metros/ y no se le quitaba la/ pero no me espanté oiga usted < usté>

$400 \mathrm{E}$ : fijese

401 I: no me espanté/ y los muchachos nomás los ú-/ lo único que les dije "itraigan una cobija!" / y luego pasan corriendo a traer la cobija/ y luego este lo fueron a tapar (entrevista 104)

[Hablando sobre los Reyes Magos]

(14) $564 \mathrm{~F}$ : sí/ usted ya no les da/ ya están bien grandes/ [secundaria]

565 I: [pero sí <-sí:> / todavia] el año pasado/ todavía <-toavía> le compré al// que patines / que bicicleta// todavía les compré

$566 \mathrm{~F}$ : fijese

567 I: pero pues <-pus> [ya <-ya:>] (entrevista 37)

En (13) y (14), las intervenciones constituidas únicamente por fijese no aportan nada al desarrollo temático de la conversación, ni tampoco presentan contenido proposicional alguno; más bien, son contribuciones de carácter estructural, cuya función fática está centrada en el canal comunicativo, por lo que pueden ser prescindibles. En este sentido, fijese está cercano a 
fórmulas como $\mathrm{mh}$, ajá, etcétera, que pueden ser parafraseadas como "sigue" o "continúa" y que han sido descritas como "respuestas fáticas: que confirman los papeles comunicativos con reacciones como ya ya, $\mathrm{mm}$ o manifiestan interés por lo que dice el interlocutor (a menudo con carácter agradador)" (Grupo Val.Es.Co., 2014: 21-2). Precisamente por esto, el interlocutor puede retomar la conversación en el mismo punto en el que la dejó, en (13) se manifiesta con la repetición de "no me espanté" antes y después del marcador, mientras que en (14) el interlocutor concluye la argumentación iniciada en el turno previo. Ahora bien, como valor secundario, y asociado a su carácter modalizante, también puede señalar la importancia de la información dada por el interlocutor. Finalmente, cabe destacar que la aparición de fijate/fijese como intervención completa fue común en el corpus, a diferencia de lo que sucedió con oye o mira, que en ningún caso aparecieron en esta posición.

\subsection{INTRODUCTOR DE DISCURSO DIRECTO CITADO}

Cuando el marcador aparece a inicio de acto, pero no de intervención, cumple con una función delimitadora, muy parecida a la de pues, en los casos donde "parcela y realza ciertas partes del discurso [y en algunos casos] se presenta en el interior de los relatos dentro de una conversación al marcar las intervenciones de los distintos personajes (una marca del paso de estilo indirecto al estilo directo, acompañado o no de un verbo de decir)" (Briz, 1993b: 43).

(15) 415 I: pero empiezan las broncas// se los voy a platicar rápido/ dice "fijese que me eché una"/// porque es muy exigente <-etsigente>/ G (entrevista 101)

(16) 178 I: y entonces// pues una vez estuv-/ estuvieron a punto de violarme/ y él fue el que este// el/ al que yo le confié "fijate que así y asi”" ¿no?/ porque viviamos en una vecindad (entrevista 95)

(17) 54 I: mi certificado y todo/ y este y ya no fui/ pero al día siguiente fui y me presenté con el doctor y le expliqué <-espliqué> / le digo "fijese que pasó esto" / le digo "por eso no vine, pero no sé si pueda darme trabajo todavia" (entrevista 57)

Asi pues, fijjate/fïjese funciona como marca de introducción de discurso directo citado, esto es, aparece en la frontera con el estilo directo ${ }^{9}$ (Pons, 1998), ya que le permite al hablante seleccionar un nivel de detalle para moldear sus ideas y destacar ciertos aspectos de la narración con lo que hace su historia más comprensible o emocionante, por lo que desempeña un papel de intensificador de la narración (Penn, 2007). Finalmente, en estos casos, el marcador está asociado a funciones conectivas, específicamente

\footnotetext{
9 Corresponde a lo que recientemente Borreguero (2017: 80 ) ha denominado función de marcas citativas.
} 
a la ordenación, señalando así que trabaja en un nivel macroestructural de segmentación del discurso.

\subsection{CAMBio de TÓPICo}

Otra función relacionada con la delimitación y ordenación del discurso es la que se presenta cuando este marcador introduce un cambio de tema; así, en estos casos, el llamado de atención señala la transición de tópico en la conversación. Con esta función, suele presentarse en el interior de intervención y se constituye como subacto adyacente textual (SAT):

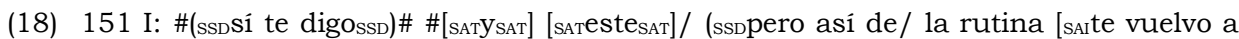
repetir $_{\text {saI }}$ / no/ no tuve mucho margen de/ de andar en la calle/ no no nossD)/ (ssxpa-

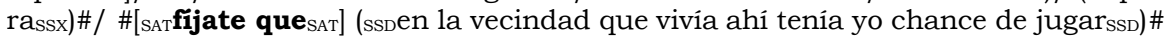
(entrevista 100)

(19) 712 I: \#(ssD nossD)\# \#[SATYSAT] (ssDsí me/ me gusta/ me gusta manejar ${ }_{\mathrm{SSD}}$ )\#

$713 \mathrm{E}:$ \#(ssDटंsí?ssD)\#

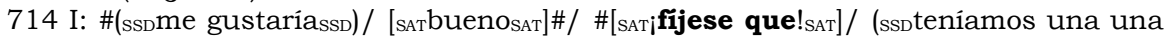
combissD)\#

715 E: \#ajá\#

716 I:\#(ssDuna combi ya viejitassD)/\# \#[SATYSAT] (ssDesa [saTeste SAT $_{\mathrm{SAT}}$ // la agarraba [sAT este $_{\mathrm{SAT}}$ ] mi papássD)\# (entrevista 83).

En (18) lo que señala el marcador es un cambio de tema, que pasa de "no tuve margen de andar en la calle" a "la vecindad y la oportunidad de jugar". En tanto que en (19) la transición va de "el gusto por manejar" al microrrelato sobre "una combi". Al igual que en los casos de introductor de discurso directo citado, el marcador funciona en un nivel macroestructural, en particular con una función conectiva de ordenador discursivo.

Hasta aquí, hemos visto la gran movilidad que puede tener este marcador dentro de las unidades del discurso, así como la relación que esto tiene con el tipo de función que desempeña; así pues, posición y función se muestran como criterios indispensables a considerar cuando se realiza un análisis de este tipo. Este hecho se ve reflejado, entonces, en las seis distintas funciones que puede cumplir. En la siguiente Tabla se presentan resumidos estos rasgos:

\begin{tabular}{|c|c|c|}
\hline Función & Posición y unidad & Valor \\
\hline Enfocador de alteridad & $\begin{array}{c}\text { Inicio (intervención / acto) } \\
\text { Intermedio (intervención / acto) }\end{array}$ & Interpersonal \\
\hline $\begin{array}{c}\text { Inicio y final (intervención / } \\
\text { acto) }\end{array}$ & Modal \\
\hline $\begin{array}{c}\text { Introductor de intervención } \\
\text { reactiva }\end{array}$ & $\begin{array}{c}\text { Inicio (intervención) } \\
\text { Social }\end{array}$ & Estructural \\
\hline $\begin{array}{c}\text { Intervención con valor fático } \\
\text { Introductor de discurso directo } \\
\text { citado }\end{array}$ & Inicio (intervención) & Textual (conexión) \\
\hline Cambio de tópico & Inicio (diálogo) & Textual (conexión) \\
\hline
\end{tabular}

Tabla: Funciones de fijate/fijese como marcador discursivo 


\section{Conclusiones}

En este estudio hemos intentado caracterizar las funciones de fijate/ fijese como marcador del discurso. El primer aspecto a destacar es la libertad que tiene para aparecer en posición inicial de diálogo, intervención y acto, interior de intervención y acto o a final de intervención y acto; esta movilidad le permite cumplir con distintos roles en diferentes niveles. Aunado a esto, el marcador presenta matices funcionales, dependiendo de cuál es la unidad pertinente de análisis.

Asi pues, como punto de partida, confirmamos que la función principal de este marcador está asociada con valores interpersonales (hacia la enunciación) y con valores modalizadores (hacia el enunciado), por lo que, en el primer caso, tiende a aparecer a inicio de intervención para reclamar la atención del interlocutor. Mientras que, en el segundo, además, puede ocurrir en posición inicial y final de intervención y de acto, para enfatizar la parcela discursiva que afecta, ya sea de forma anafórica o catafórica. Justamente por este valor fático interpersonal, la posición preferida del marcador es el inicio de intervención, por lo que ha adquirido un valor conversacional relacionado con la introducción de intervenciones reactivas, confiriéndole a esta unidad con un carácter social puesto que contribuye al desarrollo temático de la conversación.

Por otra parte, identificamos que existen casos donde una intervención completa está constituida únicamente por fíjate/fíjese, en donde el marcador es un acto y una intervención a la vez. Así, su valor funcional es uno interpersonal, asociado con la gestión de la interacción y, a diferencia del caso anterior, tiene un carácter estructural más que social, muy cercano a una función fática (Jakobson, 1981) y de control de contacto.

Por último, encontramos ejemplos donde fijate/fíjese parcela el discurso contribuyendo así a su ordenación. En estas circunstancias, trabaja en un nivel macroestructural, por lo que la posición que toma es la de inicio de diálogo. Esta función, a su vez, también está asociada con la delimitación, de tal forma que posee valores conectivos principalmente.

Es importante mencionar que, en todas estas funciones, el marcador conserva la herencia conativa del modo imperativo del que proviene, así que resulta muy dificil separar tajantemente su carácter interpersonal y modalizante. Precisamente por esto, este rasgo directivo está presente cuando: (i) llama la atención del oyente, (ii) refuerza una unidad discursiva (marca lingüistica de relevancia), (iii) introduce una intervención reactiva, al tiempo que justifica la opinión del hablante o presenta una respuesta despreferida, (iv) gestiona la interacción y destaca la información presentada por el interlocutor, (v) introduce el discurso citado y funciona como intensificador de la narración, y (vi) redirige la atención hacia un nuevo tópico. 
Por todo lo anterior, parece claro que tanto posición como unidad discursiva son dos criterios determinantes al analizar el tipo de función que pueden o no pueden desempeñar los marcadores discursivos. Además, al considerar estos dos aspectos en el análisis descriptivo de los marcadores, se puede destacar, de mejor manera, el carácter polifuncional de estas partículas. En resumen, si aceptamos que posición y unidad son fundamentales para atribuir funciones a los marcadores, debemos adoptar, siguiendo a Pons (1998), una aproximación categorial difusa que nos permita distinguir las sutilezas funcionales que puede tener cada marcador.

Finalmente, este trabajo representa un primer acercamiento al estudio de fijate/fijese, razón por la cual serán necesarios más estudios que permitan reforzar o profundizar estas hipótesis. En específico, dado que comparten un mismo origen, sería interesante realizar un estudio contrastivo que incluyera a oye y mira para determinar si existen diferencias funcionales y/o de frecuencia significativas.

\section{REFERENCIAS}

ALCINA, J. \& BLECUA, J. M. (1975): Gramática española, Madrid: Ariel.

BEINHAUER, W. (1991): El español coloquial, $3^{\text {a }}$ edición, Madrid: Gredos.

BORREGUERO ZULOAGA, M. (2017): "Los relatos coloquiales: partículas discursivas y polifonia", Pragmalingüistica, 25, pp. 6288.

BRIZ, A. (1993a): "Los conectores pragmáticos en español coloquial (I): su papel argumentativo", Contextos, XI(21-22), pp. 145-188.

BRIZ, A. (1993b): "Los conectores pragmáticos en español coloquial (II): su papel metadiscursivo", Español Actual, 59, pp. 39-56.

BRIZ, A. (1998): El español coloquial en la conversación. Esbozo de pragmagramática, Barcelona: Ariel.

BRIZ, A. (2000): "Las unidades de la conversación”, RILCE, 16(2), pp. 225-246.

BRIZ, A. (2006): "Unidades del discurso, partículas discursivas y atenuantes. El caso de 'no/ tienes razón", ponencia invitada en el Congreso "Discurso, interacción e identidad. Simposio Internacional en Homenaje a Lars Fant" celebrado en Estocolmo (Suecia).

BRIZ, A. \& GRUPO VAL.ES.CO. (2002): Corpus de conversaciones coloquiales, Madrid: Arcolibros.

BRIZ, A. \& GRUPO VAL.ES.CO. (2003): "Un sistema de unidades para el estudio del lenguaje coloquial", Oralia, 6, pp. 7-61.

BRIZ, A. \& S. PONS (2010): "Unidades, marcadores y posición", Loureda, O. y Acín, E. (coords.): Los estudios sobre marcadores del discurso hoy, Madrid: Arco, pp. 523-557.

BRIZ, A., PONS, S. \& PORTOLÉS, J. (eds.) (2008). Diccionario de partículas discursivas del español (DPDE), Publicación electrónica: http://www.dpde.es

CUENCA, M. J. \& MARÍN, J. M. (2000): "Verbos de percepción gramaticalizados como conectores: análisis contrastivo español-catalán", Revista Española 
de Lingüistica Aplicada, volumen monográfico, pp. 215-237.

DOMÍNGUEZ, C. L. \& ÁlVAREZ, A. (2005): "Marcadores en interacción: un estudio de marcadores en el español hablado en Mérida (Venezuela)", Revista Virtual de Estudos da Linguagem, 4, Publicación electrónica: http://www.revel.inf.br/files/artigos/revel_4_marcadores_en_interaccion.pdf

FERNÁNDEZ JAÉN, J. (2012): Semántica cognitiva diacrónica de los verbos de percepción fisica del español. Tesis doctoral. Director: José Luis Cifuentes Honrubia. Universitat d'Alacant.

FUENTES RODRÍGUEZ, C. (1990): "Apéndices con valor apelativo", Carbonero Cano, P. y Palet Plaja, M. T. (eds.): Sociolingüistica andaluza 5. Habla de Sevilla y hablas americanas, Sevi1la: Servicio de Publicaciones de la Universidad de Sevilla, pp. 171-196.

GALLARDO-PAÚLS, B. (2013): "Estructura textual y gestión informativa en hablantes con afasia", Miguel Casas (dir.) y Rocío Vela (ed.): Eugenio Coseriu, in memoriam. XIV Jornadas de Lingüística, Cádiz: Universidad de Cádiz, pp. 131-142.

GALLARDO-PAÚLS, B. \& MARTÍN JORDÀ, M. J. (2005): "Marcadores discursivos procedentes de verbos perceptivos en el discurso afásico", Revista de Investigación Lingüistica, 8, pp. 53-94.

GALUÉ, D. (2002): "Marcadores conversaciones: un análisis pragmático", Boletín de Lingüística, 18, pp. 27-48.

GRUPO VAL.ES.CO. (2014): "Las unidades del discurso oral", Estudios de Lingüística del Español, 35, pp. 13-73.
JAKOBSON, R. (1981): Ensayos de lingüistica general, Barcelona: Seix Barral.

MARTÍN BUTRAGUEÑO, P. \& LASTRA, Y. (coords.) (2011-2015): Corpus sociolingüístico de la Ciudad de México (CSCM). México: El Colegio de México, Publicación electrónica: http://lef.colmex.mx/index.php/investigaciones/corpus-sociolingueistico-de-laciudad-de-mexico-cscm

MARTIIN ZORRAQUINO, M. A. \& PORTOLÉS, J. (1999). "Los marcadores del discurso", Bosque I. y Demonte V. (eds.): Gramática descriptiva de la lengua española III, Madrid: Espasa, pp. 4051-4213.

PENN, C. (2007): "Cultural dimensions of aphasia: Adding diversity and flexibility to the equation", Ball, M. y Damico, J. (eds.): Clinical Aphasiology: Future Directions, Hove, UK: Psychology Press, pp. 221-244.

PONS, S. (1998): "Oye y mira o los 1ímites de la conexión", Martín Zorraquino, M. A. y Montolío Durán, E. (eds.): Marcadores discursivos: teoría y práctica, Madrid: Arco, pp. 213-228.

PONS, S. (2008). "La combinación de marcadores del discurso en la conversación coloquial: interacciones entre posición y función", Estudos Linguisticos/Linguistics Studies, 2, pp. 141159.

PONS, S. (2016): “Cómo dividir una conversación en actos y subactos", Bañón Hernández, A. M., Espejo Muriel, M., Herrero Muñoz-Cobo, B. y López Cruces, J. L. (eds.): Oralidad y análisis del discurso. Homenaje a Luis Cortés Rodriguez, Almeria: Editorial Universidad de Almeria, pp. 545-566.

PRESEEA. Proyecto para el estudio sociolingüistico del español de 
España y de América (2008). "Marcas y etiquetas mínimas obligatorias". Publicación electrónica: http://www.linguas.net/preseea

ROULET, E., AUCHLIN, A., MOESCHLER, J. \& RUBATTEL, C. (1985): L'articulation $d u$ discours en français contemporain, Bern: Peter Lang.

ROULET, E., FILLIETTAZ, L., GROBET, A. y BURGER, M. (2001): Un modèle et un instrument d'analyse de l'organisation $d u$ discours, Bern: Peter Lang.

SPERBER, D. \& WILSON, D. (1986): Relevance: Communication and
Cognition, Oxford: Basil Blackwell.

TANGHE, S. \& JANSEGERS, M. (2012): "Los marcadores del discurso derivados de los verbos de percepción: un estudio contrastivo español-italiano", II Coloquio Internacional: Marcadores discursivos en las lenguas románicas: un enfoque contrastivo, Buenos Aires: Universidad de Buenos Aires.

VIGARA TAUSTE, A. M. (1980): Aspectos del español hablado: aportaciones al estudio del español coloquial, España: Sociedad General Española de Librería. 